\title{
Valve Area Index
}

National Cancer Institute

\section{Source}

National Cancer Institute. Valve Area Index. NCI Thesaurus. Code C147157.

The ratio of the valve area to the body surface area. 